\title{
MORE NONEUCLIDIAN PID'S AND DEDEKIND DOMAINS WITH PRESCRIBED CLASS GROUP
}

\author{
PAUL EAKIN AND W. HEINZER
}

\begin{abstract}
Let $Z$ denote the integers, $Q$ the rationals, $X$ an indeterminate and $G$ a finitely generated abelian group. Then there is a Dedekind domain $D$ such that $Z[X] \subset D \subsetneq Q[X]$, and $D$ has class group $G$. If $G=0$ then $D$ is a noneuclidian PID.
\end{abstract}

If $D$ is a Dedekind domain, then the nonzero ideals of $D$ form an abelian group $I$ under multiplication. An obvious subgroup is $J$, the group of principal ideals. The group $I / J$ is called the class group or divisor class group of $D$. In [C] Claborn gives a method for constructing a Dedekind domain with any pre-assigned abelian group as class group. We offer here a theorem which allows one, given a finitely generated abelian group $G$, to construct a Dedekind domain with $G$ as class group. We feel that our construction offers much simpler rings than Claborn's and that their number theory is much more tractable. As evidence of this, it is easy to see that the PID's constructed here (i.e., those with class group zero) are not euclidian rings.

We refer the reader to the first few pages of Professor Samuel's Tata notes $\left[\mathbf{S}_{2}\right]$ or to Bourbaki [B] for elegant exposition on the class group. Our methods are extracted from these sources, $\left[\mathbf{S}_{1}\right]$ and $[\mathbf{A E H}]$.

In order to avoid repetition, all valuation rings are assumed to be rank one discrete. By an algebraic extension of a valuation ring $V$ we mean a valuation ring $V^{*}$ which extends $V$ and whose residue field is algebraic over that of $V$.

THEOREM. Let $K$ be a field and $K(t)$ a simple transcendental extension of $K$. Let $V_{1}, \cdots, V_{n}$ be distinct, rank one discrete valuation rings with quotient field $K$. For each $i$ let $\left\{V_{i, j}^{*}\right\}_{j=1}^{g_{i}}$ be a finite collection of distinct, algebraic extensions of $V_{i}$ to $K(t)$. Let $e_{i, j}$ denote the ramification index of

Presented to the Society, January 26, 1973 under the title Some Dedekind domains with specified class group and more noneuclidean PID's; received by the editors November 14, 1972.

AMS (MOS) subject classifications (1970). Primary 13-00, 13A15, 13B15, 13B25, 13E05, 13F15, 13F05, 13F10, 13F20.

Key words and phrases. Dedekind domain, euclidian ring, principal ideal domain, class group, Krull ring.

(c) American Mathematical Society 1973 
$V_{i, j}^{*}$ over $V_{i}$ and set

$$
D=\left(\bigcap_{i, j} V_{i, j}^{*}\right) \cap K[t] .
$$

Then $D$ is a Dedekind domain.

Set $m=\sum_{i} g_{i}$ and let $G$ denote the free abelian group of rank $m$ on the generators $P_{i, j}$. Let $L$ denote the subgroup of $G$ generated by the elements $R_{i}=\sum_{j=1}^{g_{i}} e_{i, j} P_{i, j}$ for $i=1, \cdots, n$. Then $C=G / L$ is the class group of $D$. Moreover, whenever $C=0, D$ is a ncneuclidian principal ideal domain.

Proof. Let $\pi_{i}$ be a prime element of $V_{i}$ and $s=\prod \pi_{i}$. Then $D[1 / s]=$ $K[t]$. Obviously the essential valuations of $s$ are exactly the $V_{i, j}^{*}$. Let $\Psi_{i, j}$ denote the center of $V_{i, j}^{*}$ on $D$. To see that $D$ is Dedekind, it suffices to show that the $\Psi_{i, j}$ are maximal ideals of $D$ since only maximal ideals containing $s$ are lost when one goes from $D$ to $D[1 / s]$. The fact that the $\Psi_{i, j}$ are maximal follows from the assumption that the $V_{i, j}^{*}$ are algebraic extensions of the $V_{i}$. This implies that $D / \Psi_{i, j}$ is algebraic over the field $V_{i} / \pi_{i}$ which makes it a field. To get Dedekind, one can also apply directly the result (5.7) of [AEH].

Let $\mathscr{D}$ be the group of divisors of $D$ and $\mathscr{P}$ the subgroup of principal divisors. Let $P_{i, j} \in \mathscr{D}$ be the divisor corresponding to $\Psi_{i, j}$, and let $G$ be the subgroup of $\mathscr{D}$ generated by $\left\{P_{i, j}\right\}$. Since $D[1 / s]=K[t]$ is a PID, it follows that $\mathscr{P}+G=\mathscr{D}$. Thus the class group $C=\mathscr{D} / \mathscr{P}=(G+\mathscr{P}) / \mathscr{P}=G / \mathscr{P} \cap G$. Let $R_{i}=\sum_{j=1}^{g} e_{i, j} P_{i, j}$ and let $L$ be the subgroup generated by the $R_{i}$. We claim $L=\mathscr{P} \cap G$. Containment one way is clear since $R_{i}$ is the divisor of $\pi_{i}$. Conversely suppose $R=\sum n_{i, j} P_{i, j} \in \mathscr{P}$. This asserts that there is an $\alpha \in$ $K(t)$ whose divisor is precisely $R$. But it must then be true that $\alpha \in K$ since only elements of $K$ can have value zero in all of the other essential valuations of $D$ (the $f$-adic valuations where $f$ is an irreducible member of $K[t])$. So if $v_{i}$ is the associated normed valuation of $V_{i}$ and $v_{i}\left(\alpha_{i}\right)=r_{i}$, then $R=\sum r_{i} R_{i}$. Hence $C=G / L$ which is exactly what was to be seen.

The fact that $D$ is not euclidian follows quickly from the transfinite construction on p. 289 of $\left[\mathbf{S}_{1}\right]$. Were $D$ euclidian it would have to be a PID and one could, by the adjunction of the reciprocal of one element, reduce to the case $D=V^{*} \cap K[t]$ where $V^{*}$ is an unramified, algebraic extension of $V=V^{*} \cap K$. If the residue field of $V^{*}$ is a proper extension of that of $V$, one cannot even proceed beyond the first step in the construction. If $V^{*}$ and $V$ have the same residue field, one cannot proceed beyond the $A_{w}$ step where $w$ is the first infinite cardinal. In this case, $A_{w}$ is easily seen to be $V$ and the construction cannot advance past $A_{w}$ : If $p$ is any prime of $D$ which might be in $A_{w+1} \backslash A_{w}$, then the mapping $D \rightarrow D / p$ induces an isomorphism on $V$. Since $D / p$ is a field, $V \rightarrow D / p$ cannot be surjective. 
Let $V$ be a rank one discrete valuation ring with quotient field $K$ and $K[t]$ a simple transcendental extension of $K[t]$. If $V^{*}$ is an extension of $V$ to $K[t]$, then $V^{*}$ is easily seen to have an infinite number of conjugates under the action of the group of fractional linear transformations of $K[t]$ over $K$. Thus to produce an infinite supply of algebraic extensions of $V$ of a given ramification index $e$, we need exhibit only one. Let $Q$ denote the rationals and $p$ a prime integer. Let $Z_{p}$ be the $p$-adic valuation ring of $Q$ and $\hat{Z}_{p}$ the $p$-adic integers. If $t$ is chosen to be an element of $\hat{Z}_{p}$ which has $p$-adic value one and is transcendental over $Q$, then $V=\hat{Z}_{p} \cap Q[t]$ is an unramified, algebraic extension of $Z_{p}$ to $Q[t]$. To get a ramified extension of a simple transcendental extension of $Q$, one can just extend $V$ to $Q\left[t^{1 / c}\right]$. We can thus state the following.

COROLLARY. Let $G$ be a finitely generated abelian group, $Q$ the rationals, $Z$ the integers, and $X$ an indeterminate. Then there is a Dedekind domain $D$ with class group $G$ such that $Z[X] \subset D \subsetneq Q[X]$.

Proof. By the fundamental theorem of finitely generated abelian groups, $G=Z^{(n)} \oplus Z / n_{1} \oplus \cdots \oplus Z / n_{e}$ where $Z^{(n)}$ is the free abelian group of rank $n$ and $Z / n_{i}$ is the cyclic group of order $n_{i}$ for $i=1, \cdots, e$.

Let $P_{0}, P_{1}, \cdots, P_{e}$ be distinct integral primes. Choose $\left\{V_{0, j}\right\}_{j=1}^{n_{0}+1}$ to be distinct, unramified algebraic extensions of $Z_{p_{0}}$ to $Q[t]$. Then for each $i$ from 1 to $e$ choose $V_{i}$ to be an algebraic extension of $Z_{p_{i}}$ to $Q(t)$ of ramification index $n_{i}$. By our theorem, $D=Q[t] \cap\left(\bigcap_{j} V_{0, j}\right) \cap\left(\bigcap_{i=1}^{e} V_{i}\right)$ is the desired example.

\section{REFERENCES}

[AEH] S. Abhyankar, P. Eakin and W. Heinzer, On the uniqueness of the coefficient ring in a polynomial ring, J. Algebra 23 (1972), 310-342.

[C] L. Claborn, Every abelian group is a class group, Pacific J. Math. 18 (1966), 219222. MR 33 \#4085.

[B] N. Bourbaki, Éléments de mathématique. Fasc. XXXI. Algèbre commutative. Chap. 7, Actualités Sci. Indust., no. 1314, Hermann, Paris, 1965. MR 41 \#5339.

[S $\mathbf{S}_{1}$ ] P. Samuel, About euclidean rings, J. Algebra 19 (1971), 282-301. MR 43 \#6190.

$\left[\mathbf{S}_{2}\right]-$ Lectures on unique factorization domains, Tata Institute of Fundamental Research Lectures on Math., No. 30, Bombay, 1964. MR 35 \#5428.

\section{Department of Mathematics, University of Kentucky, Lexington, Kentucky 40506}

Current address: Department of Mathematics, Purdue University, Lafayette, Indiana 47907 